\title{
La villine, un marqueur de différenciation utilisé en cancérologie
}

La villine est une protéine participant à la structure des microfilaments d'actine des microvillosités de bordures en brosse ; elle est en particulier synthétisée, précocement au cours du développement, dans les entérocytes et les cellules tubulaires rénales ainsi que dans leurs précurseurs. Les cellules cancéreuses dérivées de tissus exprimant normalement de la villine synthétisent également cette protéine. Une concentration élevée de villine sérique serait un utile marqueur de cancers du tractus gastro-intestinal.

\section{Sylvie Robine}

Chargée de recherche à l'Inserm

\section{Brigitte Dudouet \\ Pharmacienne, ingénieur de recher- che à l'Institut Pasteur}

\section{Daniel Louvard}

Directeur de recherche au Cnrs, chef de laboratoire à l'Institut Pasteur

\section{ADRESSE}

S. Robine, B. Dudouet, D. Louvard : unité de biologie des membranes, département de biologie moléculaire, Institut Pasteur, 25-28, rue du Dr Roux, 75724 Paris Cedex 15.

$\mathrm{m} / \mathrm{s} n^{\circ} 8$ vol. 3, octobre 87 a connaissance de plus en plus approfondie des molécules qui participent à l'élaboration des structures hautement spécialisées de la cellule constitue la base d'une nouvelle "passerelle " entre science fondamentale et médecine. Plusieurs exemples tirés de la littérature récente montrent, en effet, qu'il est possible d'utiliser des protéines de structure bien caractérisées sur le plan biochimique et biologique comme outils d'analyse des processus néoplasiques.

Les filaments intermédiaires sont constitués de protéines dont l'expression est spécifique d'un type cellulaire : les kératines dans les cellules épithéliales, la desmine dans les cellules musculaires, les neurofilaments dans les cellules nerveuses et la protéine fibrillaire acide dans les cellules gliales. Les techniques d'immunofluorescence qui visualisent les protéines associées aux filaments intermédiaires permettent de classer les principaux groupes de tumeurs. Chaque groupe peut être caractérisé par un type spécifique de filaments intermédiaires [1, 2, 3]. Par conséquent, la recherche des protéines constituant ces structures a permis la caractérisation rapide et précise de certaines tumeurs dont le diagnostic était délicat à établir à l'aide des techniques conventionnelles. D'autre part, l'utilisation des kératines, par exemple, comme marqueurs des cellules tumorales épithéliales permet l'identification de cellules disséminées dans les ganglions lymphatiques (qui normalement expriment la vimentine) à distance de la zone tumorale et constitue une application très importante en pathologie [2, 3].

La mise en évidence de protéines spécifiquement associées à des organites de différenciation, telle la synaptophysine, une glycoprotéine membranaire des vésicules présynaptiques des neurones ou 
des vésicules de sécrétion des cellules endocrines et neuroendocrines, constitue un autre exemple. Récemment, cette protéine a été retrouvée dans une grande variété de tumeurs neuroendocrines, indépendamment de l'expression d'autres marqueurs de différenciation de ces cellules [4]. En ce qui concerne les épithéliums, une approche similaire a été conduite en utilisant comme marqueurs les desmoplakines, protéines majoritaires des plaques desmosomales, sites d'attachement des filaments intermédiaires au niveau des desmosomes [5].

Cependant, il faut souligner plusieurs limitations aux outils actuellement disponibles : les kératines forment une famille constituée d'une vingtaine de polypeptides très similaires et dont certaines sont spécifiques d'un type d'épithélium. Leur présence dans un tissu tumoral permet une classification des carcinomes encore trop grossière compte tenu de la très grande diversité des origines cellulaires de ce type de tumeur. D'autre part, bien que les neurones et certaines cellules neuroendocrines expriment les neurofilaments, de nombreuses cellules neuro-endocrines expriment également les kératines en raison de leur caractère épithélial. Les tumeurs dérivant de ces cellules expriment soit les neurofilaments, soit les kératines et parfois coexpriment les deux classes de filaments.

Cette approche, où les connaissances fondamentales des molécules impliquées dans la différenciation cellulaire et/ou l'organisation intracellulaire (protéines de structure du cytosquelette, protéines membranaires de la surface ou des compartiments intracellulaires, etc.) sont utilisées en clinique humaine, contraste fortement avec la démarche qui a conduit ces vingt dernières années à la mise en évidence des marqueurs tumoraux. En effet ceux-ci, couramment utilisés de nos jours en pathologie cancéreuse, sont des molécules synthétisées par la cellule maligne ou par l'hôte en réponse à la présence de tissus cancéreux ; ils ont été mis à jour à la suite de recherche systématique d'antigènes de tumeur, dosables dans la circulation sanguine. Ces molécules sont produites par des types cellulaires très différents et sont des marqueurs ayant une sensibilité et une spécificité limitées [6].

Il est nécessaire de définir d'autres marqueurs de différenciation cellulaire utilisables en pathologie et possédant de plus une expression tissulaire restreinte. C'est dans cet axe de recherche que se situe l'approche que nous avons suivie et que nous rapportons ici. Pour caractériser l'épithélium digestif, une protéine, la villine, a d'abord été reconnue comme une molécule participant à la structure des microfilaments d'actine des microvillosités situées à l'apex des cellules intestinales différenciées. L'étude de l'expression et de la régulation du gène de la villine $a$ conduit خ̀ suggérer que celle-ci puisse jouer un rôle dans la pathologie digestive. Deux aspects ont été abordés : d'une part la recherche de la présence de la villine en anatomopathologie par des techniques d'immunofluorescence, d'autre part nous avons cherché à observer l'apparition de la villine dans le sérum des malades atteints de certaines affections du tube digestif et en particulier dans les cas de tumeurs malignes du côlon.

\section{La bordure en brosse des cellules intestinales}

La bordure en brosse des entérocytes (figure 1) est une structure subcellulaire très organisée, apparaissant au cours de la différenciation terminale des entérocytes. C'est un exemple remarquable d'assemblage de novo d'un organite cellulaire. Une dizaine de protéines (représentant $80 \%$ des protéines totales des microvillosités) ont été caractérisées et leur rôle précisé.

Plusieurs enzymes, appartenant à la classe des hydrolases (aminopeptidases, disaccharidases et phosphatase alcaline) et ayant un rôle dans la digestion terminale 


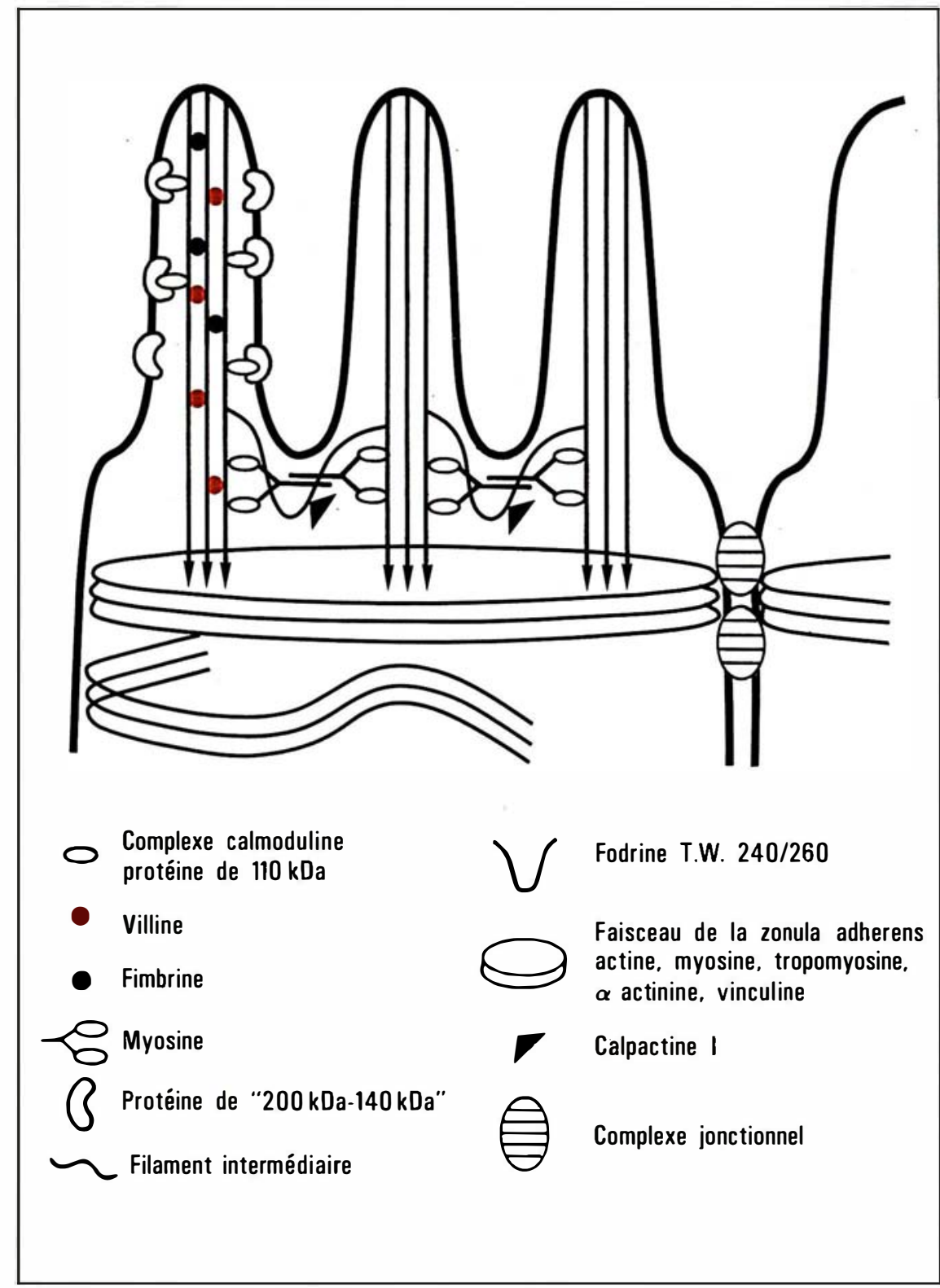

Figure 1. Modèle de l'organisation moléculaire du cytosquelette de la bordure en brosse intestinale. On distingue deux zones : les microvillosités contenant les microfilaments d'actine et de protéines associées, et la région $d^{\prime}$ ancrage des microfilaments. Dans cette zone, trois réseaux de filaments sont mis en évidence : deux d'entre eux (les filaments intermédiaires et les filaments de la zonula adherens) forment une ceinture perpendiculaire à l'axe des microfilaments contenus dans les microvillosités. Le troisième réseau forme un domaine de liaison entre les extrémités des filaments $d^{\prime}$ actine. Les protéines principales qui constituent ces réseaux sont mentionnées. La protéine de " 200 kDa-140 kDa " décrite par E. Coudrier [15] participe à l'attachement latéral des microfilaments avec la membrane des microvillosités. La fodrine ou les TW 240/260 (TW pour "terminal web "), selon les espèces étudiées, sont des protéines de la famille des spectrines qui, associées à la myosine et à la calpactine 1, participent à la liaison entre les filaments $d^{\prime}$ actine des microvillosités dans la partie apicale de la cellule [8]. Dans le complexe jonctionnel, on trouve une protéine de surface, l'uvomoruline [16], ainsi qu'une protéine associée à la face cytoplasmique de la membrane " 20-1 " [17]. Nous remercions E. Coudrier pour l'élaboration et la réalisation de cette figure.

$m / s n^{\circ} 8$ vol. 3 , octobre 87 des aliments, ont été purifiées et leur disposition topologique par rapport à la membrane de même que leur biosynthèse in vivo et in vitro ont été étudiées [7].

Jusqu'en 1978, on a considéré que les microfilaments des microvillosités intestinales étaient constitués d'actine et de protéines habituellement associées aux filaments d'actine dans les cellules non musculaires. Les travaux entrepris par les groupes de $\mathrm{K}$. Weber (Göttingen, RFA), M. Mooseker (Yale University, New Haven, USA) ainsi que les nôtres ont montré que plusieurs protéines liant l'actine in vitro et formant le cytosquelette des microvillosités étaient différentes de celles rencontrées dans d'autres types cellulaires [8].

La protéine la mieux caractérisée dans cette catégorie est sans doute la villine. Les autres protéines majoritaires formant le cytosquelette des microvillosités intestinales sont la fimbrine, une protéine de $110 \mathrm{kDa}$ et la calmoduline. Les propriétés de ces trois protéines liant l'actine sont indiquées sur la figure $2, p$. 482. Il faut noter que seule la villine est retrouvée dans un nombre très limité de tissus.

\section{Spécificité d'expression tissulaire}

La villine est une protéine de structure de $95 \mathrm{kDa}$ qui se lie à l'actine de façon dépendante du calcium. Lorsque la concentration intracellulaire de cet ion est inférieure à $10^{-7} \mathrm{M}$, la villine provoque le rassemblement des filaments d'actine en faisceaux, alors qu'elle entraîne un raccourcissement de ces filaments (figure 2) lorsque la concentration calcique est supérieure à $10^{-7} \mathrm{M}[8]$. Des anticorps polyclonaux et monoclonaux ont été préparés afin d'étudier la spécificité d'expression tissulaire de la villine. Un anticorps monoclonal a été sélectionné du fait de sa grande affinité pour la villine humaine et de son utilisation dans plusieurs conditions expérimentales, notamment en immunofluorescence, en immunolocalisation ultrastructurale et en 
LA FIMBRINE (68 kDa)

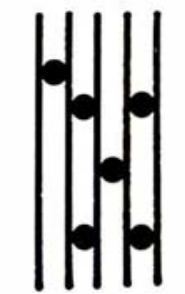

Maintient l'actine

en faisceau

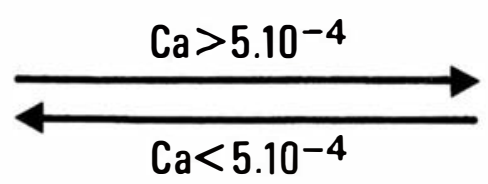

Ca $<5.10^{-4}$

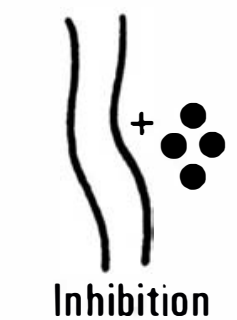

de l'interaction

actine/fimbrine

\section{LA VILLINE $(90-95 \mathrm{kDa})$}

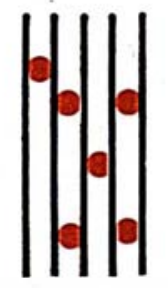

Maintient l'actine en faisceau
$10^{-6}<\mathrm{Ca}<10^{-7}$

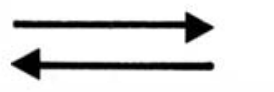<smiles>CCOC</smiles>

Raccourcissement des filaments

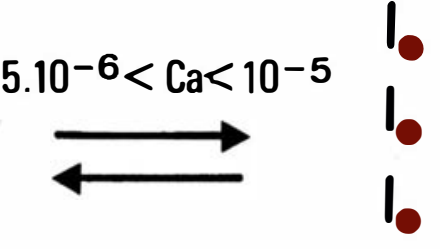

Coupure des filaments

\section{LA PROTEINE ( $110 \mathrm{kDa}$ )}

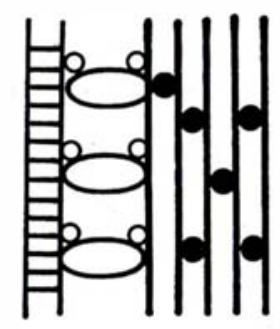

- Lie la calmoduline (0)

- Lie l'actine en absence d'ATP

- A une activité ATPase analogue à celle d'une myosine musculaire

- Interagit avec la membrane (目)

Figure 2. Propriétés de liaison à l'actine des trois protéines majeures des microvillosités intestinales. Les symboles utilisés sont les mêmes que dans la figure 1. Nous remercions E. Coudrier qui a réalisé cette figure. 
dosage immuno-enzymatique (ELISA) [9]. Au moyen de techniques d'immunofluorescence, la villine a été retrouvée chez l'homme adulte dans les cellules épithéliales ayant une bordure en brosse bien organisée et en particulier dans les cellules de transport de l'intestin grêle, du côlon et les cellules épithéliales du tubule proximal rénal (figure 3). Détectée par des techniques d'immunocytochimie ultrastructurale (marquage à l'or colloïdal), la villine a été mise en évidence dans les microvillosités intestinales. Elle est concentrée sur l'axe des microfilaments et sur leur prolongement dans le cytoplasme sous-jacent. Cette distribution s'observe aussi bien dans les cellules de l'intestin grêle que dans celles du côlon humain. Dans les deux types cellulaires, on observe également une distribution diffuse et homogène. Dans ces expériences, il n'a pas été mis en évidence d'association préférentielle avec une structure subcellulaire particulière [9].

D'autres cellules épithéliales ayant une même origine embryologique mais dépourvues de bordure en brosse expriment un taux plus faible de villine, par exemple les cellules canalaires du pancréas, les cellules des canaux biliaires principaux et les cellules de la muqueuse de l'antre pylorique (figure 3). La villine n'a pas été retrouvee dans d'autres épithéliums, en particulier au niveau des cellules de la muqueuse fundique, ni dans les hépatocytes, ni dans les cellules pancréatiques acineuses, exocrines ou endocrines. Elle est absente de tous les autres épithéliums simples ou stratifiés. Elle n'a jamais été identifiée dans d'autres types cellulaires. Ces observations indiquent le petit nombre de types cellulaires dans lesquels cette protéine est retrouvée. Plus tard, nous avons démontré la présence de cette protéine dans les cellules épithéliales indifférenciées des cryptes de la muqueuse intestinale chez l'homme adulte. De plus, la villine a été détectée dans les cellules immatures du tube digestif pri$m / s n^{\circ} 8$ vol. 3 , octobre 87 mitif, lors de l'embryogenèse, avant que la phase finale de l'histogenèse ne soit abordée (fœetus humain de huit semaines, embryon de rat de 11 jours) ([10] et J.C. Maunoury, hôpital SainteAnne, Paris, résultats non publiés). La villine permet donc l'identification des cellules indifférenciées qui, plus tard, formeront les différents tissus (rein, tube digestif) où elle est exprimée.

L'étude de la biosynthèse de la villine effectuée avec des cellules intestinales en culture a permis de montrer que les cellules indifférenciées synthétisaient moins de villine que les cellules. différenciées $[9,10]$. Cela est dû au nombre plus faible de copies d'ARN messager existant dans les cellules indifférenciées que celui observé dans les cellules différenciées [11]. Ces résultats suggèrent que la villine est non seulement un marqueur qui caractérise l'origine d'une cellule, mais aussi un marqueur permettant d'apprécier, au niveau moléculaire, le stade de différenciation des cellules capables d'exprimer le gène codant pour cette protéine.

\section{Un marqueur tissulaire de lésions cancéreuses}

A la suite des recherches concernant l'expression tissulaire de la villine et constatant sa présence dans un nombre limité d'épithéliums du tractus gastro-intestinal et uro-génital, les questions fondamentales liées à l'identification d'un marqueur moléculaire pour caractériser l'origine tissulaire de foyers néoplasiques ont été abordées. Nous avons d'abord mis en évidence le maintien d'expression de la villine dans les adénocarcinomes coliques indépendamment
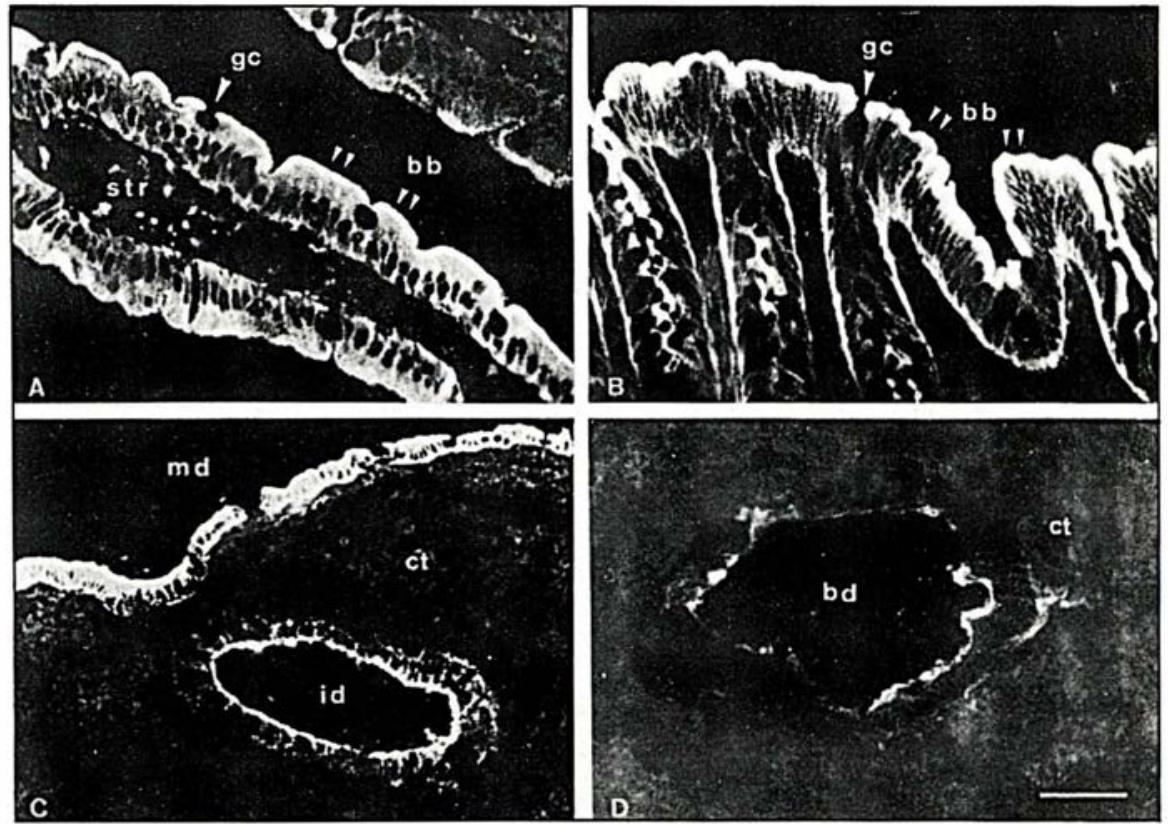

Figure 3. Expression tissulaire de la villine. (A) intestin grêle de rat. (B) côlon de rat : les bordures en brosse (bb) sont très fortement marquées; on observe un marquage diffus dans le cytoplasme des cellules absorptives; par cette technique, la villine n'est pas mise en évidence dans les cellules à mucus (gc). (C) pancréas humain : la villine est observée uniquement dans les cellules des canaux principaux (md) ou dans les cellules des canaux interlobulaires (id) ; le tissu conjonctif est négatif. (D) foie humain : les canaux biliaires principaux expriment la villine; le parenchyme hépatique est négatif; barre $=0,5 \mu \mathrm{m}$. (Tiré de [10]). 


\section{RÉFÉRENCES}

9. Dudouet B, Robine S, Huet C, et al. Changes in villin synthesis and subcellular distribution during intestinal differenciation of HT29-18 clones. J Cell Biol 1987; 105 : 359-70.

10. Robine S, Huet C, Moll R, et al. Can villin be used to identify malignant and undifferentiated normal digestive epithelial cells ? Proc Natl Acad Sai USA 1985 ; 82 : 8488-92.

11. Pringault E, Arpin M, Garcia A, Finidori J, Louvard D. A human villin cDNA clone to investigate the differenciation of intestinal and kidney cells in vivo and in culture. EMBO $J 1986$; 5 : 3119-24.

12. Moll R, Robine S, Dudouet B, Louvard D. Villin, a cytoskeletal protein and an early differenciation marker that is expressed in a limited array of human adenocarcinomas. Virchows Arch [Pathol Anat] (sous presse).

13. West $\mathrm{AB}$, Isaac $\mathrm{CA}$, Carboni JM, Morrow JS, Mooseker MS, Barwick KW. Localization of villin, a cytoskeletal protein specific to microvilli, in human ileum and colon and in colonic neoplasms. Gastroenterology (sous presse).

14. Grōne $\mathrm{HJ}$, Weber $\mathrm{K}$, Helmchen $U$, Osborn M. Villin. A marker of brush border diffenciation and cellular origin in human renal cell carcinoma. Am $J$ Pathol $1986 ; 124$ : du stade de différenciation morphologique au sein de la tumeur étudiée [10]. Puis, récemment, le Dr. R. Moll, de l'université de Mayence (RFA), en collaboration avec notre laboratoire, a réalisé une étude exhaustive de l'expression de la villine dans des adénocarcinomes localisés dans un grand nombre de tissus [12]. Plusieurs conclusions fondamentales peuvent en être tirées :

- L'expression de la villine est toujours maintenue dans les processus néoplasiques des tissus épithéliaux qui, normalement, l'expriment : il s'agit des adénocarcinomes coliques et rectaux $[12,13]$, de ceux dérivant du tubule proximal rénal $[12,14]$ et des adénocarcinomes du pancréas [12]. La situation inverse est aussi vérifiée : les adénocarcinomes de la glande mammaire ou de l'ovaire n'expriment pas plus la villine que les épithéliums sains présents dans ces deux organes. - La localisation de la protéine au pôle apical des cellules est conservée dans les cellules tumorales différenciées.

- L'expression spécifique et uniforme de la villine dans les tubules d'un adénocarcinome est retrouvée dans les ganglions lymphatiques et les métastases à distance.

- La villine est retrouvée dans les adénocarcinomes coliques peu différenciés lorsqu'il y a formation de structures tubulaires étroites, même rudimentaires (figure 4).

- Cependant, il existe quelques exceptions ; en particulier le docteur R. Moll a mis en évidence la présence de villine dans certains adénocarcinomes de l'endomètre (4/10), de l'ovaire (1/9) et du poumon (4/18). Les observations histologiques semblent exclure l'hypothèse d'une situation métastatique. On observe un marquage apical discret d'un petit nombre de foyers hétérogènes qui contraste avec le marquage apical intense, uniforme, observé dans les autres adénocarcinomes (côlon, rein, pancréas). Cette différence quantitative et qualitative du marquage observé permet une distinction nette pour l'interprétation des coupes. L'expression de la villine, même occasionnelle, dans les tumeurs provenant de tissus adultes normaux qui ne l'expriment pas pose un problème fondamental. Les hypothèses qui permettraient d'expliquer la ré-expression de la villine dans ces tumeurs sont en cours d'étude (embryogenèse, précurseur des étapes néoplasiques, etc.).

- Enfin, tous les adénocarcinomes de l'estomac où l'on observe la formation de tubules expriment fortement la villine alors que seules les cellules normales de la muqueuse de l'antre pylorique sont faiblement positives.

En conclusion, la présence de la
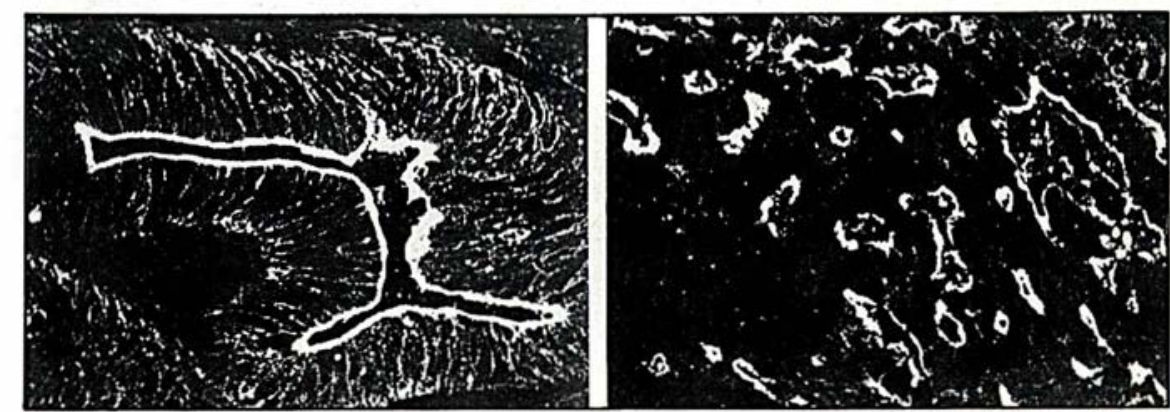

Figure 4. Immunolocalisation de la villine dans les adénocarcinomes coliques. Le cliché de gauche montre un adénocarcinome colique différencié, le cliché de droite, un adénocarcinome colique peu différencié avec formation de tubules rudimentaires. (Tiré de [12]). 
villine dans un nombre limité d'épithéliums permet d'envisager son utilisation dans le diagnostic anatomopathologique.

\section{Détection dans le sérum} en pathologie digestive

Les différentes observations indiquées ci-dessus ont confirmé le maintien de l'expression de la villine lors du processus néoplasique, quel que soit l'état de différenciation des tumeurs qui l'expriment. Les adénocarcinomes étant le siège de processus physiopathologiques provoquant des lésions (nécrose, ulcération, inflammation), il est possible d'imaginer que la villine est libérée dans la circulation sanguine à la suite de lyse cellulaire, le relargage de cette protéine pouvant être facilité par la vascularisation très développée de certains carcinomes.

$\mathrm{Ce}$ travail concerne uniquement les affections du tractus gastrointestinal. Les maladies rénales, en cours d'étude, ont été volontairement exclues.

Grâce à un test immuno-enzymologique très sensible permettant de détecter jusqu'à $0,5 \mathrm{ng}$ de villine par millilitre de sérum, nous avons mis en évidence la villine dans les sérums de patients atteints de tumeurs malignes du tube digestif. Afin de vérifier que l'immunodétection de la villine est bien significative d'un cancer du tractus gastro-intestinal, une étude a été menée dans une population générale constituée de 418 donneurs sans trouble digestif déclaré. 93,3\% des donneurs ne présentaient aucune trace de villine circulante; $4,3 \%$ montraient une concentration sérique comprise entre 0 et $20 \mathrm{ng} / \mathrm{ml}$ et $2,4 \%$ de la population avaient une concentration de villine s'échelonnant entre 20 et $100 \mathrm{ng} / \mathrm{ml}^{*}$. Après examen de ces résultats, la concentration dé base a été fixée à 20 nanogrammes/ml.

\footnotetext{
* Il n'a pas été possible, pour des raisons d'éthique, de recontacter ces 10 personnes, donneurs de sang dans un centre de transfusion sanguine, afin de réaliser des examens complémentaires.

Ces recherches ont été poursuivies dans des cas de maladies digestives bénignes. Sur 46 cas examinés (polyposes coliques, maladies de Crohn, rectocolites hémorragiques, ulcères gastriques), une concentration anormale de villine sérique n'a été trouvée que trois fois. Cependant, le nombre de sérums analysés est encore insuffisant.

La villine a également été dosée dans 40 observations de cancers extra-digestifs. Dans 39 cas (cancers du sein, de l'ovaire, du poumon, myosarcome, etc.) les résultats étaient négatifs. Cela confirme la spécificité d'expression tissulaire de cette molécule et le maintien de celle-ci au cours du processus néoplasique.

Parallèlement, une étude concernant les tumeurs digestives malignes a été menée. L'échantillonnage dans chaque catégorie de cancers digestifs est pour le moment encore limité, mais il semble que la détection de la villine soit particulièrement intéressante dans les cas de cancers colorectaux dont 42 cas ont été étudiés. $52 \%$ de ces cancers étaient détectés au stade B de la classification de Dukes** et près de $70 \%$ au stade C. Les concentrations de villine sérique retrouvées dans ces cas s'échelonnaient entre 40 et $3000 \mathrm{ng} / \mathrm{ml}$. De plus, toutes les carcinoses péritonéales d'origine colique étaient positives, contrairement à celles d'origine différente.

Certains cancers du pancréas ont montré une concentration élevée de villine circulante $(300 \mathrm{ng} / \mathrm{ml})$. Il s'agit vraisemblablement de tumeurs malignes ayant pour origine les cellules canalaires pancréatiques, cellules qui expriment la villine à l'état normal. D'autre part, dans les cancers gastriques associés à une métaplasie intestinale, on observe des concentrations pathologiques de villine sérique.

\footnotetext{
* Classification de Dukes. Stade A : tumeur limitée à la paroi intestinale; stade $B$ : tumeur envahissant la séreuse sans atteindre les ganglions lymphatiques; stade $C$ : tumeur envahissant les ganglions régionaux; stade $D$ : présence de mélastases da distance.
}

D’après ces résultats préliminaires, il semble que la villine soit un marqueur de lésions tissulaires spécifiques de pathologies malignes du tractus gastro-intestinal. Nous proposons l'hypothèse suivante : la lyse cellulaire induite par les phénomènes de nécrose, d'inflammation et/ou d'ulcération provoquerait une libération de la villine dans la lumière intestinale dans le cas des maladies bénignes, alors que cette protéine serait relarguée dans le milieu intérieur au niveau du réseau capillaire assurant l'apport nutritif des cellules malignes.

\section{Perspectives cliniques}

Plusieurs points en cours d'étude doivent être précisés : en particulier, il est important de définir si la villine peut être utilisée dans le dépistage précoce des cancers digestifs et si, dosée parallèlement aux autres marqueurs tumoraux, elle peut être un complément efficace dans l'établissement d'un diagnostic ; enfin, son intérêt dans le suivi post-opératoire des cancers digestifs reste à établir.

Dans les traitements chimiothérapiques provoquant la lyse des cellules néoplasiques, il est possible d'imaginer que cette protéine puisse être libérée au cours du traitement et être ainsi un témoin de l'efficacité de cette thérapeutique.

Un fait important a été observé : dans les cas d'ulcères gastriques avec métaplasie intestinale, la villine a été retrouvée aussi bien en immunocytochimie dans le tissu métaplasique (docteur R. Moll) que dans les sérums, et cela à des concentrations pouvant atteindre plusieurs centaines de nanogram$\mathrm{mes} / \mathrm{ml}$. Ces résultats réclament une confirmation sur un plus grand nombre de cas, mais présagent de l'intérêt d'une telle observation, les métaplasies intestinales pouvant évoluer vers un état cancéreux.

D'autre part, la présence de villine dans les métastases hépatiques de tumeurs coliques primitives suggère qu'elle pourrait être un moyen de dépistage et de surveil- 
lance des métastases, ainsi qu'un élément intervenant dans la décision d'effectuer ou non une exérèse.

Enfin, les techniques de radiomarquage des anticorps monoclonaux anti-villine et leur utilisation en scintigraphie isotopique pourraient permettre de localiser la tumeur, d'évaluer l'étendue de l'invasion cancéreuse et de mettre en évidence d'éventuelles micrométastases.

\section{Conclusion}

Les résultats rapportés sur l'étude de l'expression de la villine au cours de la différenciation normale des entérocytes chez l'adulte montrent que cette protéine de structure, associée aux microfilaments d'actine des microvillosités intestinales, est exprimée dans un nombre limité de types cellulaires du tractus gastro-intestinal et uro-génital. La villine est présente dans les cellules qui possèdent une bordure en brosse bien caractérisée (intestin, rein, tube afférent du testicule); elle est aussi exprimée dans des cellules différenciées sans bordure en brosse qui dérivent du tube intestinal primitif (canaux pancréatiques et biliaires, cellules de l'antre pylorique). L'étude détaillée au cours de l'histogenèse précoce de l'ébauche intestinale (qui se situe vers le $8-10^{e}$ jour chez l'embryon de rat) est en cours, en collaboration avec le docteur J.-C. Maunoury de l'hôpital Sainte-Anne à Paris. Les résultats déjà obtenus illustrent l'apparition précoce de cette protéine dans les cellules embryonnaires, précurseurs des cellules qui, chez l'adulte, expriment la villine. Ainsi avons-nous pu mettre en valeur les propriétés d'une nouvelle protéine du cytosquelette qui possède une spécificité d'expression restreinte et dont la biosynthèse est maintenue au cours des processus néoplasiques. Son utilisation en anatomopathologie a été bien cernée ; son intérêt en biologie clinique est prometteur mais nécessite des études complémentaires

\section{Summary}

Villin is an actin binding protein associated with microfilaments bundles of microvilli forming brush borders. Villin is expressed in a few normal differentiated and undifferentiated epithelial cells of gastrointestinal and urogenital tracts.

We have studied its expression in different human epithelia and in corresponding carcinoma at different stages of malignancy. Results of these investigations have shown that the tissue specific expression of villin is maintained during neoplasic processes even when tumors are poorly differentiated (metastasis). Its detection (using immunocytochemical techniques performed with antibodies against villin) together with conventional morphological criteria and other tissue specific markers such as proteins of intermediate filaments (e.g. : keratin and vimentin) allow to establish or to confirm an histodiagnosis for the origin and type of the primary tumor.

Furthermore a sensitive quantitative ELISA test has been developed using monoclonal antibodies, to measure villin in human sera. Preliminary studies have shown that there is a positive correlation between the amount of villin in sera and clinical diagnosis of gastrointestinal cancer. This protein is not detected in the large majority of normal blood donors, or in sera of patients with benign digestive diseases, or in the case of nondigestive cancers. Therefore, a serologic diagnosis using villin may prove to be a useful biological test to identify a digestive cancer or for the follow-up of patients after surgery.

S. Robine : unité de biologie des membranes, département de biologie moléculaire, Institu

Pasteur, 25-28, rue du Dr Roux, 75724 Paris Cedex 15. 